\title{
IMPACTO DE LA COVID-19 EN LAS PERSONAS CON DISCAPACIDADES INTELECTUALES Y DEL DESARROLLO, SUS FAMILIARES Y LOS PROFESIONALES Y ORGANIZACIONES DE APOYO
}

\section{Impact of COVID-19 on people with intellectual and developmental disabilities, their families, support professionals and organizations}

Manuela Crespo CuAdrado

Universidad de Salamanca. Instituto Universitario de Integración en la Comunidad

Miguel Ángel Verdugo Alonso

Universidad de Salamanca. Instituto Universitario de Integración en la Comunidad

Patricia Navas Macho

Universidad de Salamanca. Instituto Universitario de Integración en la Comunidad

Sergio Martínez Torres

Sociólogo

Antonio Manuel Amor GonzÁlez

Universidad de Salamanca. Instituto Universitario de Integración en la Comunidad

Recepción: 23 de marzo de 2021

Aceptación: 5 de mayo de 2021

Resumen: Nuestro mundo se ha visto transformado por el coronavirus y la infección que provoca. La gravedad de la enfermedad y las consecuencias del estado de alarma no afectan a todos por igual, siendo las personas con discapacidad intelectual y del desarrollo uno de los colectivos más vulnerables. El INICO y Plena inclusión realizaron un estudio con la finalidad de analizar el impacto de la COVID-19 y el confinamiento durante la primera ola en 2020 en las personas con discapacidad intelectual y del desarrollo, 
IMPACTO DE LA COVID-19 EN LAS PERSONAS CON DISCAPACIDADES INTELECTUALES

Y DEL DESARROLLO, SUS FAMILIARES Y LOS PROFESIONALES Y ORGANIZACIONES DE APOYO

M. CRESPO, M. Á. VERDUGO, P. NAVAS, S. MARTÍNEZ, A. M. AMOR

sus familiares, personal de atención directa y organizaciones. Para ello, se elaboraron y aplicaron en España cuatro encuestas en línea, participando cerca de 1.500 personas. Los resultados indican que, a pesar del esfuerzo realizado por el tercer sector, la crisis sanitaria ha dejado al descubierto las carencias de nuestro sistema de apoyos, lo que lleva a reflexionar sobre tres aspectos fundamentales: el grado en que se trabaja en el desarrollo y mantenimiento de redes naturales de apoyo cuando la persona vive en un entorno específico; la medida en que los apoyos ofrecidos desde el tercer sector llegan a contextos que se encuentran fuera de la red de servicios ofertados por el movimiento asociativo; y el papel de nuestro sistema educativo a la hora de garantizar el derecho a la educación de personas con necesidades especiales de apoyo.

Palabras clave: COVID-19; confinamiento; discapacidad intelectual y del desarrollo; familiares; profesionales de atención directa; gerentes o responsables de servicios; apoyos; derechos.

Aвstract: Our world has been transformed by the coronavirus and the infection it causes. The severity of the disease and the consequences of the state of alarm do not affect everyone equally, being people with intellectual and developmental disabilities one of the most vulnerable groups. INICO and Plena inclusion conducted a study to analyze the impact of COVID-19 and lockdown on people with intellectual and developmental disabilities, their families, direct support professionals, and disability organizations. To address this goal, four online surveys were developed and completed by almost 1.500 participants from all the Spanish regions. The results indicate that, despite the efforts made by the third sector, the health crisis has unveiled the deficiencies of our support system, which leads us to reflect on three fundamental aspects: the degree to which the development and maintenance of natural support networks is carried out when the person lives in a specific environment; the extent to which the support offered from the third sector reaches individuals outside the disability services network; and the role of our educational system in guaranteeing the right to education for people with special support needs.

KeYwords: COVID-19; lockdown; intellectual and developmental disabilities; family members; direct care professionals; service managers; supports; rights.

\section{Introducción}

$\mathrm{E}$

L MUNDO EN EL QUE VIVIMOS se ha visto drásticamente transformado por el coronavirus SARS-CoV-2 y la infección que provoca, la COVID-19, siendo España uno de los países más afectados. A fin de combatir dicha infección, el 14 de marzo de 2020 el Gobierno de España decretó el estado de alarma por emergencia sanitaria (Real Decreto 463/2020), cuya vigencia se prolongó hasta el 21 de junio de 2020 (Real Decreto-Ley 21/2020).

Aunque la amenaza de infección es real para todas las personas, la gravedad de la enfermedad y las consecuencias derivadas del estado de alarma no afectan a todos por igual, siendo las personas con discapacidades intelectuales y del desarrollo (DID) uno

Ediciones Universidad de Salamanca / CC BY-NC-ND

Siglo Cero, vol. 52, número extraordinario, 2021, pp. 11-36 
de los grupos poblacionales especialmente vulnerables a esta situación (Courtenay y Perera, 2020; Naciones Unidas, 2020), debido a que tienen mayor probabilidad de ser infectadas por el virus y sufrir cuadros más graves de la enfermedad (Tummers et al., 2020). Además, en estas personas el riesgo y la severidad de la infección pueden verse incrementados por posibles comorbilidades (Naciones Unidas, 2020; Organización Mundial de la Salud, 2020) y porque, en ocasiones, viven en entornos residenciales con una alta concentración de personas, lo cual dificulta el mantenimiento de la distancia física, especialmente entre aquellos que requieren un apoyo más intenso (SaftaZecheria, 2020).

En España se estima que son más de 30.000 las personas con DID que viven en entornos residenciales (Navas et al., 2017) y que, por tanto, se ven expuestas a un mayor riesgo de contagio. En estos contextos dependen, en mayor o menor medida, de apoyos profesionales que, en el contexto de la COVID-19, se han visto significativamente reducidos o alterados adoptando, en ocasiones, formas de provisión de difícil acceso para las personas con necesidades de apoyo más extensas (Navas et al., 2021). Por los motivos expuestos se aprecia la necesidad de analizar el impacto de la COVID-19 sobre la recepción de apoyos.

A esto hay que añadir que las organizaciones del tercer sector no han recibido, por parte de las administraciones responsables, los apoyos necesarios para hacer frente a esta crisis sanitaria (European Association of Service Providers for Persons with Disabilities EASPD, 2020), lo que lleva a la necesidad de analizar cómo esta carencia de apoyos ha influido en la vida de las personas con DID, sus familias y los profesionales de apoyo. Por otra parte, la pandemia también ha puesto de manifiesto la fragmentación existente entre servicios sociales y sanitarios, otorgando a los primeros responsabilidades y funciones correspondientes a estos últimos (Organización Mundial de la Salud, 2020), y todo sin contar con medidas de protección suficientes y en un contexto de provisión de apoyos que arrastra déficits en cuanto a recursos materiales y humanos.

Las medidas sanitarias adoptadas durante el estado de alarma en España se han intensificado y han adquirido un carácter mucho más restrictivo en los servicios de apoyo a las personas con discapacidad intelectual y del desarrollo, lo cual ha tenido consecuencias sociales y psicológicas para este colectivo que conviene analizar. Además, muchos han visto sus rutinas interrumpidas al tener que dejar de acudir a sus centros y servicios (Hughes y Anderson, 2020; Plena inclusión, 2020a). A esto se añade que a muchas personas con DID, especialmente las que tienen más necesidades de apoyo (en España unas 63.000, según Navas et al., 2017), les ha sido difícil comprender la situación y autorregular su conducta. Esto aumenta el riesgo de aparición de conductas desafiantes que afecten negativamente a su bienestar emocional y su calidad de vida (Courtenay, 2020; Courtenay y Perera, 2020), así como a la de sus familiares quienes, en muchos casos, han asumido en exclusividad los apoyos que requieren estas personas (Rose et al., 2020).

La conjunción de la pandemia y las medidas aplicadas para combatirla suponen una amenaza para los derechos de las personas con DID (Naciones Unidas, 2020), más aún si tenemos en cuenta que los cuidados de larga duración (Organización Mundial 
ssde la Salud, 2020) y, de manera particular, la provisión de apoyos a personas con discapacidad intelectual no han estado presentes en el debate sobre las medidas a adoptar en el contexto de la COVID-19 (EASPD, 2020). Las dificultades de los servicios para ofrecer apoyos adecuados a las personas con DID en este contexto han dado lugar a familias sobrecargadas que han tenido que suplir esta carencia de apoyos profesionales, en ocasiones sin contar con las herramientas necesarias, al no ser posible, en muchos casos, el apoyo domiciliario (Courtenay, 2020). Estas dificultades se han hecho especialmente patentes en el ámbito escolar (Esentürk, 2020; Jeste et al., 2020; Navas et al., 2020). Ello hace necesario analizar el impacto de la COVID-19 en las familias debido a la sobrecarga de cuidados.

En estos meses, la investigación sobre el SARS-CoV-2 y la COVID-19 se ha multiplicado, especialmente en el ámbito biomédico, con especial énfasis en el desarrollo de una vacuna que prevenga el contagio (e. g., Folegatti et al., 2020) y en tratamientos efectivos para combatir la COVID-19 (e. g., Beigel et al., 2020). Sin embargo, a pesar de que la literatura científica subraya la mayor vulnerabilidad de las personas con DID en diferentes ámbitos de su vida, las instituciones sanitarias no han proporcionado datos sobre el impacto de la COVID-19 en este colectivo.

Por los motivos expuestos, desde el Instituto Universitario de Integración en la Comunidad de la Universidad de Salamanca (INICO), en colaboración con Plena inclusión, se planteó llevar a cabo un estudio con la finalidad de analizar el impacto de la COVID-19 en las personas con discapacidad intelectual y del desarrollo, sus familiares, el personal de atención directa y apoyo y las organizaciones del tercer sector, centrándonos de manera particular en el periodo de confinamiento experimentado durante los meses de marzo a junio de 2020 (para más información consultar Navas et al., 2020). Es de destacar la alianza permanente entre INICO y Plena inclusión para accionar de forma proactiva y conjunta iniciativas de investigación vinculadas a las necesidades urgentes de las personas y de las familias.

\section{Metodología}

\subsection{Instrumento ${ }^{1}$}

Teniendo en cuenta este objetivo, se elaboraron cuatro encuestas, aplicadas en línea en España, y dirigidas a los principales grupos implicados: personas con discapacidades intelectuales y del desarrollo; familiares y/o tutores legales; profesionales de atención directa; y gerentes o responsables de servicios. En el caso de la encuesta dirigida a personas con DID, y para facilitar la participación de todas las personas, se recogió la posibilidad de que el familiar o profesional contestara a la misma en calidad de "traductor vital" si la persona presentaba necesidades muy extensas de apoyo que dificultaran la cumplimentación autónoma, o con ayuda, del cuestionario.

\footnotetext{
1 https://inico.usal.es/analisis-del-impacto-y-seguimiento-de-la-emergencia-covid-19-en-poblacion-con-discapacidad-intelectual-y-del-desarrollo-en-espana/
} 
La elaboración de las encuestas partió de la revisión de la literatura científica sobre el coronavirus y su impacto en la salud y en las condiciones de vida de las personas con discapacidad; de los informes y directrices elaborados por organismos internacionales (e. g., EASPD, OMS, ONU) sobre los potenciales riesgos que plantea la pandemia; y de los repositorios y webs institucionales que recopilan información sobre la COVID-19 y las personas con DID. Además, se partió del modelo de calidad de vida de Schalock y Verdugo (2002) para incluir preguntas que permitieran conocer el impacto de la situación sanitaria en las ocho dimensiones que configuran una vida de calidad para todas las personas.

Tras el análisis de estas fuentes, el equipo de investigación elaboró las encuestas preliminares, que fueron enviadas a un equipo de siete expertos en materia de discapacidad intelectual y del desarrollo, procedentes del ámbito de investigación, de la atención directa y gestión de organizaciones proveedoras de apoyos y servicios, y compuesto por profesionales del campo de la medicina, el derecho, la psicología y la sociología. Se discutieron las preguntas a incorporar en cada encuesta, así como su redacción y organización en secciones, y los cuestionarios dirigidos a las personas con DID y sus familias se enviaron a familiares y personas con discapacidad para que valorasen la pertinencia de los mismos e hiciesen las puntualizaciones que estimasen oportunas. Por último, la encuesta dirigida a personas con DID fue redactada y validada a lectura fácil por el equipo de accesibilidad de Plena inclusión.

\subsection{Procedimiento}

Las encuestas fueron enviadas por INICO y Plena inclusión a diferentes organizaciones nacionales del ámbito de la discapacidad intelectual y del desarrollo, y se difundieron también a través de sus respectivas redes sociales. Este procedimiento fue revisado y aprobado por el comité ético de Plena inclusión España.

$\mathrm{L}$ a recogida de datos tuvo lugar entre el 1 y el 30 de junio de 2020. Hay que tener en cuenta que, debido a la situación de confinamiento, fue necesario aplicar la encuesta en formato digital, lo que puede haber dejado "fuera" del estudio a aquellas personas con mayores necesidades de apoyo y/o dificultades especiales de comunicación y acceso a la tecnología que no hayan podido contar con la ayuda de un familiar o profesional en calidad de "traductor vital". La encuesta, creada a partir de la aplicación Lime Survey, se alojó en el servidor de la Universidad de Salamanca con el objetivo de maximizar la privacidad de los datos.

\subsection{Análisis de datos}

Todas las encuestas incluyen preguntas cerradas y abiertas. El análisis de las respuestas de los participantes a las preguntas cerradas se ha llevado a cabo a través del cálculo de estadísticos descriptivos (i. e., frecuencias y porcentajes). Además, se exploró, mediante test chi-cuadrado, la existencia de dependencia estadística entre 
las características sociodemográficas de los participantes (e. g., presencia de grandes necesidades de apoyo, edad, entorno residencial) y las respuestas dadas a las preguntas sobre el impacto del confinamiento. Se calcularon los tamaños del efecto de las asociaciones objeto de estudio mediante el coeficiente $V$ de Cramer y se siguió la clasificación habitual de los tamaños del efecto para este estadístico (Akoglu, 2018), según la cual: $V>, 25$ indica un efecto muy fuerte, $V>, 15$ fuerte, $V>, 10$ moderado, $V>, 05$ débil y $V<, 05$ despreciable. Los datos se analizaron utilizando IBM Statistical Package for the Social Sciences (SPSS) versión 25.

También se analizaron la mayor parte de los comentarios que las personas con DID, sus familiares y organizaciones y profesionales que prestan apoyos escribieron en las preguntas abiertas. Después de haber leído las respuestas de los participantes en varias ocasiones, el análisis de contenido (i. e., la codificación) implicó la organización de los datos en categorías o temas de interés tratados por los encuestados (Baralt, 2011). Todas las categorías se crearon de manera inductiva, sin tratar de encajarlas en un marco de codificación preexistente (Pope et al., 2005). Las categorías fueron creadas por dos de los autores de este artículo y revisadas por el equipo de investigación. Las dudas menores en la codificación o categorización fueron discutidas por el equipo de investigación hasta alcanzar un consenso.

Con el objetivo de explorar posibles diferencias individuales a la hora de responder a estas preguntas abiertas, los datos cualitativos también se han transformado en datos cuantitativos (se consideró una categoría como presente -1 - o ausente $-0-$ si esta aparecía en la respuesta del participante o no, respectivamente) (Sandelowski et al., 2009). Dado que cuando se utilizan preguntas abiertas dentro de una encuesta algunas personas pueden optar por no responder (O’Cathain y Thomas, 2004), este análisis nos permitirá reflejar el número de encuestados que expresaron una determinada opinión en las preguntas abiertas formuladas.

\subsection{Participantes}

En este estudio han participado casi 1.500 personas procedentes de todas las comunidades y ciudades autónomas de España: 582 personas con discapacidades intelectuales y del desarrollo; 323 familiares y/o tutores legales; 495 profesionales; y 75 responsables o gerentes de centros y servicios.

\subsubsection{Personas con discapacidades intelectuales y del desarrollo}

Las personas con discapacidad intelectual y del desarrollo que participaron en el estudio $(\mathrm{N}=582)$ tenían edades comprendidas entre 3 y 83 años $(M=35,6$ años; $D T$ $=14,1)$, superando la mayor parte los 21 años. Más del $60 \%$ de los participantes estudiaban y/o trabajaban antes del confinamiento y residían en el hogar familiar (Tabla 1). Hay que señalar que casi dos tercios contestaron directamente a la encuesta, ya fuera de forma autónoma $(40,9 \%)$ o con apoyo de otra persona $(23,2 \%)$. 


\begin{tabular}{|c|c|c|}
\hline Variable & Frecuencia & Porcentaje \\
\hline \multicolumn{3}{|l|}{ Sexo } \\
\hline Hombre & 279 & 47,9 \\
\hline Mujer & 303 & 52,1 \\
\hline \multicolumn{3}{|l|}{ Grupo de edad } \\
\hline Hasta los 21 años & 84 & 14,4 \\
\hline Entre 22 y 44 años & 344 & 59,1 \\
\hline Mayores de 45 años & 154 & 26,5 \\
\hline \multicolumn{3}{|l|}{ Entorno de vivienda } \\
\hline Casa propia & 63 & 10,8 \\
\hline Hogar familiar & 401 & 68,9 \\
\hline Vivienda de grupo & 55 & 9,4 \\
\hline Residencia & 51 & 8,8 \\
\hline Otro lugar & 12 & 2,1 \\
\hline \multicolumn{3}{|l|}{ Ocupación } \\
\hline Estudiando & 135 & 23,2 \\
\hline Trabajando & 195 & 33,5 \\
\hline Estudiando y trabajando & 42 & 7,2 \\
\hline No estudia ni trabaja & 210 & 36,1 \\
\hline Total & 582 & 100 \\
\hline
\end{tabular}

\subsubsection{Familiares y tutores legales}

Los familiares de las personas con DID que respondieron a la encuesta $(\mathrm{N}=323)$ fueron principalmente mujeres $(73,7 \%)$, siendo los progenitores en la mayor parte de los casos $(73,9 \%)$, especialmente las madres $(\mathrm{N}=179)$. El 43,7 \% ostentaban, además, la condición de tutor legal de la persona con DID. La edad de los familiares y tutores legales osciló entre 26 y 83 años $(M=52,3 ; D T=10,5)$, teniendo el $41,8 \%$ entre 41 y 55 años (Tabla 2). Es importante destacar que casi el $90 \%$ de las familias participantes presentaba unas condiciones económicas favorables. 


\begin{tabular}{|l|c|c|}
\hline \multicolumn{2}{|c|}{ TabLa 2. Características de los familiares y/o tutores legales } \\
\hline Variable & Frecuencia & Porcentaje \\
\hline Relación de parentesco & 67 & 20,1 \\
\hline Padre & 179 & 53,8 \\
\hline Madre & 26 & 7,8 \\
\hline Hermano & 49 & 14,7 \\
\hline Hermana & 12 & 3,6 \\
\hline Otros & $333^{*}$ & 100,0 \\
\hline Total & 3 & 0,9 \\
\hline Tutor legal & 141 & 43,7 \\
\hline Solo tutor & 179 & 55,4 \\
\hline Familiar/tutor & 323 & 100,0 \\
\hline Solo familiar & 34 & 10,5 \\
\hline Total & 135 & 41,8 \\
\hline Edad & 113 & 35,0 \\
\hline De 25 a 40 años & 41 & 12,7 \\
\hline De 41 a 55 años & 323 & 100,0 \\
\hline De 56 a 65 años & & \\
\hline Mayores de 65 años & & \\
\hline Total & & \\
\hline
\end{tabular}

*Nota: La suma total de los casos en la variable 'relación de parentesco' es de 333 personas ya que en ocho casos el cuestionario fue cumplimentado por varios familiares.

Un 84,8 \% indicaron que su familiar pasó el confinamiento con ellos y, además de la persona con DID, casi el 40 \% de las familias tenían a otra persona a su cargo. La edad de las personas con DID que habitaban en estos hogares osciló entre 2 y 73 años $(M=29,8 ; D T=15,3)$, si bien el 78,3 \% era mayor de 18 años. El 62,5\% de la muestra estuvo integrada por hombres y un $54,5 \%$ presentaba grandes necesidades de apoyo.

\subsubsection{Profesionales}

El 84,6 \% de los trabajadores encuestados (un total de 495) desempeñaban su actividad profesional en alguna organización vinculada a Plena inclusión, y ocho de cada diez eran mujeres (79,4 \%). Su edad osciló entre 20 y 64 años $(M=39,3 ; D T=9,7)$, el

Ediciones Universidad de Salamanca / CC BY-NC-ND

Siglo Cero, vol. 52, número extraordinario, 2021, pp. 11-36

$$
-18-
$$


62,1 \% con edades comprendidas entre 31 y 50 años (Tabla 3). La media de experiencia profesional fue de casi 10 años y prestaban apoyo a un total de 19.267 personas con discapacidades intelectuales y del desarrollo.

\begin{tabular}{|l|c|c|}
\hline \multicolumn{2}{|c|}{ Tabla 3. Características de los profesionales } \\
\hline Eariable & Frecuencia & Porcentaje \\
\hline Edad & 109 & 22,0 \\
\hline Hasta 30 años & 178 & 36,0 \\
\hline De 31 a 40 años & 129 & 26,1 \\
\hline De 41 a 50 años & 79 & 16,0 \\
\hline Más de 50 años & 495 & 100,0 \\
\hline Total & 77 & 15,6 \\
\hline Años de experiencia & 110 & 22,3 \\
\hline Menos de 2 años de experiencia & 188 & 38,1 \\
\hline De 2 a 5 años de experiencia & 119 & 24,1 \\
\hline De 5 a 15 años de experiencia & $494 *$ & 100,0 \\
\hline Más de 15 años de experiencia &
\end{tabular}

*Se desconoce el dato en un caso.

\subsubsection{Responsables o gerentes de centros y servicios}

Los datos obtenidos muestran que el $88,0 \%$ de los cuestionarios recibidos recogieron la situación de entidades que forman parte de Plena inclusión España. El 56,0 \% de las encuestas fueron contestadas por gerentes de organizaciones (o de federaciones en dos casos), de los cuales nueve eran a su vez responsables de un servicio o centro. La edad de los responsables y gerentes que participaron en el estudio osciló entre 30 y 68 años $(M=47,8 ; D T=8,8)$, estando la mitad de las edades comprendidas entre 41 y 50 años (Tabla 4). Su experiencia profesional oscilaba entre los seis meses y los 40 años, si bien el 75,6 \% llevaban trabajando en el sector más de ocho años $(M=15,5$; $D T=9,4)$ y solo un $6,8 \%$ tenían una experiencia profesional inferior a dos años. 


\begin{tabular}{|c|c|c|}
\hline Variable & Frecuencia & Porcentaje \\
\hline \multicolumn{3}{|l|}{ Profesión } \\
\hline Gerente de la organización o entidad & 33 & 44,0 \\
\hline Responsable del servicio o centro & 33 & 44,0 \\
\hline $\begin{array}{l}\text { Gerente de la organización/entidad y responsable del } \\
\text { servicio o centro }\end{array}$ & 9 & 12,0 \\
\hline Total & 75 & 100 \\
\hline \multicolumn{3}{|l|}{ Experiencia profesional } \\
\hline Hasta 2 años de experiencia & 5 & 6,8 \\
\hline De 3 a 7 años de experiencia & 13 & 17,6 \\
\hline De 8 a 15 años de experiencia & 24 & 32,4 \\
\hline Más de 15 años de experiencia & 32 & 43,2 \\
\hline Total & $74 *$ & 100,0 \\
\hline \multicolumn{3}{|l|}{ Edad } \\
\hline De 30 a 40 años & 15 & 20,0 \\
\hline De 41 a 50 años & 34 & 45,3 \\
\hline De 51 a 60 años & 19 & 25,3 \\
\hline Más de 60 años & 7 & 9,3 \\
\hline Total & 75 & 100,0 \\
\hline
\end{tabular}

*Se desconoce el dato en un caso.

El 35,6 \% de la muestra se compuso de entidades de pequeño tamaño, que no llegaron a superar los 100 usuarios; el 27,4\% estuvo integrado por organizaciones que apoyan a un número de personas con DID comprendido entre 100 y 200; y el $37,0 \%$ pueden ser consideradas de gran tamaño, al prestar apoyo a más de 200 personas con DID.

\section{Resultados}

3.1. Impacto de la COVID-19 y el confinamiento en las personas con discapacidad intelectual y del desarrollo y sus familias

Un $89,5 \%$ de las personas con DID manifestaron haber recibido información sobre el coronavirus, siendo fácil de entender en la mayoría de los casos $(81,0 \%)$, aun- 
que los menores de 21 años han tenido más dificultades (39,7 \%) (Amor et al., 2021). Esta información ha sido proporcionada fundamentalmente por las organizaciones del tercer sector $(46,6 \%$ ) y las familias $(35,5 \%)$, siendo residual el papel de centros escolares, laborales y sanitarios. Por su parte, el $75 \%$ de las familias consideran esencial la información sobre cómo apoyar a la persona con DID, y la han recibido en el $75 \%$ de los casos, especialmente por parte de la organización.

3.1.1. Prevalencia de la COVID-19 en personas con discapacidades intelectuales y del desarrollo

Los datos proporcionados por las familias y las personas con DID que participaron en el estudio muestran una prevalencia de un $5 \%$ de la COVID-19 en las personas con DID, porcentaje similar al arrojado por los estudios de seroprevalencia en nuestro país, lo que explica que el $95 \%$ no tuviera que acudir a los servicios sanitarios por motivos de salud relacionados con la COVID-19. Son pocas las personas con DID contagiadas y son pocas las que han podido hacerse la prueba, especialmente si convivieron en el hogar familiar durante el confinamiento: aproximadamente, solo una de cada cuatro pudo hacerse un test, siendo las que residían en entornos residenciales dependientes de organizaciones las que más pudieron hacerse la prueba $(67,9 \%)$. Esto pudo deberse a que, según los datos ofrecidos por las organizaciones, en casi la mitad de las organizaciones hubo algún contagio, por lo que al $80 \%$ de los profesionales y personas con DID vinculados a los centros y servicios les hicieron el test.

\subsubsection{Cambios en el lugar de residencia y ocupación}

La COVID-19 y el periodo de confinamiento han impactado en las condiciones de vida de las personas con DID y sus familias. En relación con su lugar de residencia, el $79,7 \%$ de las personas con DID que participaron en el estudio pasaron el confinamiento en su hogar o con su familia y el 9,8\% tuvieron que cambiar de domicilio, implicando este cambio, en la mayor parte de los casos, el desplazamiento del contexto residencial o de vivienda dependiente de una organización al hogar familiar.

Casi la mitad de las personas que estudiaban, un $46,3 \%$, tuvieron dificultades para continuar con el curso escolar, destacando las dificultades para comprender las explicaciones del profesor y las tareas $(61,0 \%)$, los problemas en su atención/ concentración o de interacción a través de los medios online $(7,3 \%)$ y la falta de apoyos $(7,3 \%)$. Un 58,8 \% recibieron algún tipo de ayuda, sobre todo de la familia $(82,6 \%)$, para continuar con su educación a distancia, especialmente los menores de 21 años $(80,9 \%)$. Por su parte, el 70,9\% de los participantes con discapacidad intelectual y del desarrollo que trabajaban no pudieron seguir haciéndolo durante el confinamiento. 
Hay que señalar que, aunque las familias participantes presentaban unas condiciones económicas favorables, un 22,8 \% consideraron que, por diferentes motivos, su situación económica ha empeorado y han visto reducidos sus ingresos a causa de la situación provocada por la COVID-19.

\subsubsection{Efectos del confinamiento en las personas con discapacidades intelectuales y del desarrollo}

A pesar de las dificultades a las que se han tenido que enfrentar las personas con DID y sus familiares, un $85,1 \%$ de las familias indican que la persona con DID se adaptó bien a la situación de confinamiento, aunque esta adaptación fue algo más difícil si la persona presentaba grandes necesidades de apoyo debido, fundamentalmente, a los cambios en la rutina, la dificultad para comprender la situación y el dejar de recibir algunos servicios.

Se le ha privado de todo lo que tenía, y él no lo entiende. No tiene alternativas online al no mirar el ordenador. Se han olvidado de las personas con más necesidades de apoyo y $\sin$ alternativas.

El cambio de rutinas y de no socialización con otros niños en la escuela le ha provocado alteraciones del sueño, del humor y cierta regresión en hábitos adquiridos.

Esta buena adaptación de la persona con DID se debe, según las familias, al esfuerzo que han realizado para seguir con las rutinas de la persona y ofrecerle los apoyos necesarios, y a la oportunidad que ha supuesto el confinamiento para pasar más tiempo juntos, pero también a la capacidad de la persona para adaptarse a los cambios y/o comprender la situación. Este trabajo realizado por las familias en sus hogares ha sido destacado por los profesionales y los responsables de las organizaciones.

Mi hijo es un niño pequeño y disfruta mucho de la compañía de sus padres. El confinamiento ha significado más tiempo de disfrute con ambos progenitores.

Es una persona que suele adaptarse bien a los cambios y para ella este ha sido uno más.

Para facilitar la mejor adaptación de las personas con DID a la situación de alarma sanitaria y, en consecuencia, la de los familiares con los que convivían, el movimiento asociativo solicitó al Gobierno la posibilidad de que las personas con discapacidad pudieran salir a la calle a través de las llamadas "salidas terapéuticas”. En relación con esto, los datos recogidos reflejan que un 78,1 \% hizo uso de ellas.

A pesar de esta buena adaptación, la emergencia sanitaria y el confinamiento han tenido consecuencias importantes en las condiciones de vida de todas las personas, y tanto las personas con discapacidades intelectuales y del desarrollo como las familias y los profesionales coinciden en señalar el bienestar emocional y las relaciones interpersonales como las dimensiones de calidad de vida más afectadas (Figura 1). 


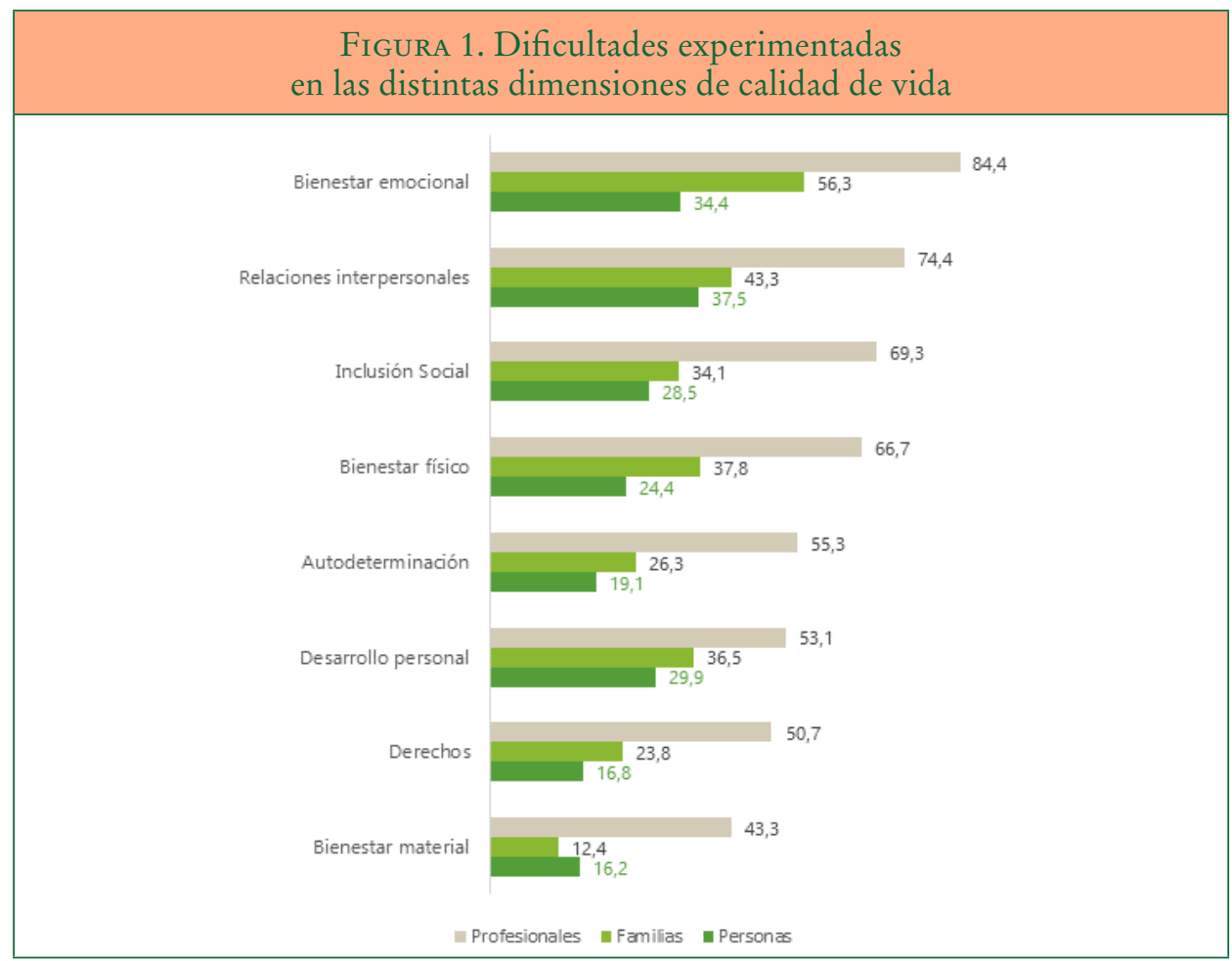

A nivel emocional, destaca el incremento de la ansiedad y el miedo en más de la mitad de los participantes: el 59,8\% manifestó haber sentido miedo por el coronavirus y el 59,6\% estar más nervioso, independientemente de su edad, contexto de residencia u ocupación. Además, las familias indicaron que los problemas de conducta aumentaron en el $37 \%$ de los participantes con DID que ya los presentaban; y un $43,9 \%$ retrocedieron en habilidades ya adquiridas. Todo ello llevó a estas familias a situaciones frecuentes de desgaste emocional: un $66,3 \%$ de los familiares que pasaron el confinamiento con la persona con DID experimentaron más estrés o ansiedad debido a la sobrecarga de cuidados y las dificultades para conciliar vida familiar y laboral, especialmente si tenían a otra persona a su cargo en el hogar o convivieron con una persona con necesidades de apoyo extensas.

El 74,5\% de los participantes han echado de menos, sobre todo, el contacto con familiares y amigos; el 45,1 \%, las actividades recreativas o de ocio; y el $20 \%$, los servicios y apoyos profesionales específicos. Cabe destacar que el 36,7\% de los estudiantes menores de 21 años echaron de menos su actividad diaria, cifra que se reduce al 20,7 \% entre los que trabajaban. Los mayores de 44 años que residían en servicios vinculados a una organización lo que más echaron en falta fue la realización de actividades recreativas o de ocio (56,4\%), así como el contacto con familiares o amigos 
$(85,5 \%)$, en comparación con sus iguales residentes en el hogar familiar o en su propia casa (25,6 \% y 68,3 \% respectivamente). En las personas con DID que residían en los centros residenciales, la situación se complicó y se vieron más afectadas sus relaciones personales al sufrir más restricciones y más intensas durante el confinamiento y a las dificultades de acceso a herramientas tecnológicas.

\subsubsection{Cambios experimentados en la recepción de apoyos y servicios habituales}

El 82,1 \% de las personas con DID afirmaron haber recibido algún apoyo durante el confinamiento, siendo los principales el de la familia $(72,8 \%)$ y las organizaciones proveedoras de servicios o sus profesionales (37,0 \%). Un $20 \%$ afirmó no haber contado con los apoyos profesionales que necesitaba, siendo la mayor parte de este porcentaje personas con DID residiendo en el hogar familiar.

El contexto de residencia ha condicionado el tipo de apoyo recibido: aquellos que residían en contextos específicos parecen haber contado con menos apoyos naturales y quienes convivieron con su familia dependieron en gran medida de los apoyos proporcionados por esta $(83,5 \%)$.

El apoyo por parte de los centros educativos a quienes estaban estudiando ha sido anecdótico: tan solo un 13,2 \% de los estudiantes menores de 21 años mencionaron haber recibido apoyo de los profesores o del centro educativo, lo que ha llevado a la familia a una sobrecarga al tener que adoptar diferentes roles para garantizar el bienestar de la persona con DID.

En las familias, las situaciones de estrés se intensifican al haberse reducido significativamente los apoyos profesionales y/o haber cerrado servicios esenciales para ellas, lo que les ha llevado a adoptar diferentes roles para asegurar el bienestar de su familiar con DID. Un 51,8 \% tuvieron dificultades para prestar los apoyos que la persona con DID necesitaba, especialmente si tenía grandes necesidades de apoyo $(65,1 \%)$. Debido al escaso apoyo recibido de los centros educativos, tres de cada cuatro familias de menores de 21 años tuvieron dificultades para que su hijo siguiera el curso escolar; tres de cada cuatro familias dejaron de recibir algún servicio o experimentaron cambios significativos en su prestación; y una de cada tres familias necesitó ayuda adicional, principalmente respiro y apoyo emocional, para prestar los apoyos necesarios a su familiar con DID durante el confinamiento, sobre todo si este tenía grandes necesidades de apoyo, pero solo la mitad de las familias recibieron dicha ayuda.

E1 74,3\% de las familias indican haber dejado de recibir o haber experimentado algún cambio en al menos uno de los servicios que recibía la persona con DID: el 68,7 \% indicaron que los servicios seguían prestándose a distancia; un 31,3\% vieron reducidos estos apoyos de manera significativa, afectando esta reducción especialmente a los servicios de apoyo en centros educativos (56,5\%) y atención temprana (44,4 \%); y el resto dejó de recibir por completo los servicios con los que contaba como, por ejemplo, los servicios de ocio y el centro de día.

Las familias de las personas con DID que pasaron el confinamiento en servicios residenciales señalaron que estas recibieron los apoyos necesarios y valoraron positivamente 
la actuación de las organizaciones durante la crisis sanitaria, aunque una de cada dos familias manifiestó haber mantenido una comunicación menos frecuente de lo habitual con su familiar con DID residente en un centro.

Es importante señalar que las personas con DID no solo han recibido apoyos, sino que también los han proporcionado: algo más de la mitad de los participantes $(51,5 \%)$ afirma haber prestado apoyo a personas de su entorno durante el confinamiento, principalmente a familiares $(55,0 \%)$, compañeros de vivienda $(25,3 \%)$ y amigos $(22,3 \%)$, siendo este apoyo de carácter lúdico (41,8\%) y emocional $(31,8 \%)$, principalmente. Las personas que han residido en servicios dependientes de una organización han sido un pilar fundamental para sus compañeros durante el confinamiento $(70 \%)$.

A mis padres, haciendo la compra para ellos. A los amigos por videoconferencia.

A los compañeros. Intento que no se peleen, pero no es fácil, no hacen mucho caso y discuten mucho.

También hay que destacar que, a pesar de las dificultades señaladas, el 57,3\% de las familias manifestaron que el clima familiar no se vio negativamente afectado durante el confinamiento e incluso mejoró para un $14,6 \%$.

\subsubsection{Preocupaciones de cara al futuro}

Un 78,6 \% de las familias encuestadas se mostraron preocupadas por cómo la crisis de la COVID-19 afectará al futuro de la persona con DID, especialmente por cómo se adaptará la persona al contexto de "nueva normalidad" y posible convivencia con el virus; por la continuidad de los apoyos y servicios tal y como han sido hasta el momento conocidos; y por los efectos que la situación de confinamiento y COVID-19 ha tenido o tendrá en la salud física y mental de la persona con DID. Estas preocupaciones se acentúan en las familias de personas con grandes necesidades de apoyo $\left(\mathrm{X}^{2} 1,323=8,3 \mathrm{p}<0,01 ; \mathrm{V}=0,16\right)$.

Tan solo un tercio de las familias consideraron que desde las distintas entidades públicas (estatales, autonómicas y locales) se han tomado medidas suficientes para proteger la vida y la seguridad de las personas con DID y el 61,1 \% de las familias encuestadas afirmaron que la situación provocada por la COVID-19 ha puesto de manifiesto la necesidad de realizar cambios en los centros y servicios para prestar mejores apoyos a las personas con DID. Estos cambios no se refieren a la actuación profesional, que valoran positivamente, sino, mayoritariamente, a la necesidad de mejorar las ratios de profesionales-usuarios para dar apoyos más personalizados; flexibilizar la prestación de apoyos y reducir las grandes estructuras que concentran a un gran número de personas; e introducir medidas que refuercen su seguridad y protección. Además, sería necesaria una mayor formación e implicación de las familias y reducir la brecha digital en las personas con discapacidades intelectuales y del desarrollo. 


\subsection{Impacto de la COVID-19 y el confinamiento en los profesionales de atención directa}

El 83,6 \% de los profesionales afirmaron haber contado con información suficiente sobre cómo apoyar a las personas con DID durante el periodo de confinamiento, sobre todo por parte del centro o servicio en el que desarrollaban sus funciones $(62,0 \%)$ y de Plena inclusión (45,5 \%). Además, un 55,3 \% participaron en distintas acciones formativas llevadas a cabo desde la Confederación, cuya calidad fue valorada muy positivamente.

\subsubsection{Prevalencia}

Los datos aportados por los profesionales que siguieron desarrollando su actividad durante el periodo de confinamiento revelan que la prevalencia de la COVID-19 en este grupo fue similar a la observada en la población general, de acuerdo con los últimos estudios sobre seroprevalencia: un 2,9\% de los profesionales han padecido la enfermedad y un 2,7 \% han sido considerados como caso posible de infección. Es preciso destacar que el porcentaje de positivos fue mayor entre aquellos que desarrollaron su trabajo de manera presencial frente a los que teletrabajaron $(4,8 \%$ frente a $1,5 \%)$. No obstante, solo la mitad pudo hacerse el test.

\subsubsection{Cambios en las condiciones laborales}

La situación provocada por la COVID-19 ha producido importantes cambios en las condiciones laborales de los profesionales del tercer sector que han afectado a las personas con DID y sus familias. Durante el confinamiento, los profesionales encuestados han prestado apoyo a 19.267 personas con discapacidades intelectuales y del desarrollo, y solo la cuarta parte permanecieron en su puesto o servicio habitual, siendo el 70,2 \% personal vinculado a centros residenciales. La mitad de los profesionales tuvieron que dejar su puesto de trabajo presencial para teletrabajar, siendo el servicio de apoyo en centro educativo ordinario el que ha tenido mayores dificultades en este sentido; un $7,7 \%$ se vieron afectados por un expediente de regulación temporal de empleo o perdieron su puesto de trabajo, lo que los llevó a problemas de tipo emocional $(71,1 \%)$; y un $13,5 \%$ experimentaron cambios significativos en sus funciones o servicio de trabajo (e. g., reubicación en otro servicio).

Debido a estos cambios solo el 31,5\% de las personas con DID a quienes estos profesionales apoyaban siguieron recibiendo sus apoyos de manera presencial durante el confinamiento; el 60,0 \% lo hicieron de forma telemática; y el 8,5\% se vieron afectados por el despido o ERTE de sus profesionales (Tabla 5). Estos datos explican que tres de cada cuatro familias dejaran de recibir algún servicio o experimentaran cambios significativos en su prestación. 
Tabla 5. Personas con DID y apoyos recibidos durante el confinamiento por parte de los profesionales que responden a la encuesta

\begin{tabular}{|l|c|c|}
\hline \multicolumn{1}{|c|}{ Personas con DID y apoyos recibidos } & N. ${ }^{\circ}$ usuarios & $\%$ \\
\hline Total usuarios que recibían apoyos antes del confinamiento & 19.267 & 100,0 \\
\hline $\begin{array}{l}\text { Personas que recibían apoyos por profesionales que ahora } \\
\text { están en situación de ERTE o despido }\end{array}$ & 1.652 & 8,5 \\
\hline Total usuarios cuyos profesionales siguen en activo & 17.615 & 91,5 \\
\hline Total usuarios que reciben apoyos presenciales & 6.063 & 31,5 \\
\hline Total usuarios que reciben apoyos a distancia & 11.552 & 60,0 \\
\hline
\end{tabular}

El 26,6 \% de los profesionales no pudieron prestar los apoyos de manera telemática debido, principalmente, a la dificultad o no disponibilidad de acceso a las nuevas tecnologías de las personas con DID; y casi el $80 \%$ de los que realizaban el trabajo de manera presencial han visto modificadas las tareas y funciones que desarrollaban en su día a día. Sin embargo, hay que señalar que, a pesar de estas dificultades, familiares, responsables y gerentes de organizaciones y las propias personas con DID valoran muy positivamente el trabajo realizado por los profesionales del tercer sector.

Los profesionales que han desarrollado su actividad de manera presencial han tenido más dificultades que los que han trabajado de manera telemática: el 23,4\% indicaron que tuvieron dificultades que incidieron en el desempeño de sus funciones (dificultades para conciliar vida familiar y laboral, sectorización de espacios, focalización en tareas de desinfección...); y un tercio considera que el material de protección disponible fue insuficiente.

Debido a las dificultades en su trabajo, los profesionales han vivido situaciones de desgaste emocional: tres cuartas partes del conjunto de profesionales encuestados han visto incrementados sus niveles de estrés y ansiedad debido sobre todo a la sensación de 'impotencia' al no poder desarrollar su trabajo como desearían, y uno de cada tres temió por su salud en su puesto de trabajo, incrementándose el número entre aquellos que trabajaron en entornos residenciales $(55,3 \%)$.

\subsubsection{Percepción de los profesionales del impacto del confinamiento en las personas} con discapacidad intelectual y del desarrollo

El 77,8 \% de los profesionales que siguieron en activo durante el confinamiento consideran que este ha tenido consecuencias negativas en las personas con DID, siendo las más frecuentes el incremento de nervios o ansiedad, tristeza, incremento de problemas de conducta y deterioro de habilidades ya adquiridas. A pesar de esto, la mayor parte de los profesionales $(94,9 \%$ ) consideran que las personas con DID se adaptaron bien o muy bien a la situación de confinamiento, destacando el papel que ha jugado la familia en ello. 
Un $75,8 \%$ de los profesionales consideran que las personas con DID recibieron los apoyos necesarios, aunque el resto manifiestan que estos apoyos han sido insuficientes, debido, principalmente, al olvido por parte de la Administración de las personas con discapacidad y al cierre de muchos servicios que no fueron considerados como esenciales para estas personas, y que hubo que prestar de manera telemática.

Aun con las restricciones sanitarias, el 78,2 \% de los profesionales que siguieron trabajando durante el confinamiento afirman que pudieron seguir prestando apoyos teniendo en cuenta los deseos y preferencias de las personas con DID, aunque casi un $10 \%$ consideran que la situación de alarma sanitaria ha dado lugar a que se produzcan situaciones no éticas en la prestación de apoyos, como son la prohibición de visitas en los contextos residenciales o la implantación de aislamientos prolongados en el tiempo.

\subsubsection{Preocupación de los profesionales por el futuro}

A pesar de que la mayoría de los profesionales que han seguido en activo, especialmente los que teletrabajaron, valoraron de manera positiva la respuesta de su centro o servicio ante la situación provocada por la COVID-19, casi un $40 \%$ indicaron que necesitaron apoyos instrumentales y emocionales con los que no contaron. Sin embargo, no valoraron igual la gestión del Gobierno y las comunidades autónomas para proteger a las personas con DID, ya que más del $60 \%$ de los profesionales encuestados consideraron que dicha gestión podría haber sido mejor (más medidas de protección, protocolos más específicos...).

La situación vivida ha dado lugar a que el 63,6 \% de estos trabajadores consideren necesario realizar modificaciones en los servicios y centros actuales, destacando la necesidad de avanzar hacia la prestación de apoyos en contextos naturales; la transformación tecnológica de las organizaciones y la mejora del acceso a posibles apoyos telemáticos; la adopción de modelos centrados en la persona y su familia, y la mejora de la ratio de profesionales-usuarios. A pesar de esto, un $70 \%$, sobre todo los que trabajaron telemáticamente, afirman que el confinamiento ha mejorado algunos aspectos de su trabajo, especialmente la posibilidad de ofrecer apoyos de manera continuada a través de la tecnología; la colaboración con las familias y la posibilidad de prestar apoyos más individualizados a quienes residieron en el hogar familiar; una mayor flexibilidad en las condiciones de trabajo; y la oportunidad para reinventarse y plantear la transformación de servicios.

\subsection{Impacto de la COVID-19 y el confinamiento en las organizaciones del tercer sector}

\subsubsection{Impacto de la COVID-19 en la situación económica de las entidades participantes}

Las organizaciones han realizado un esfuerzo económico considerable para garantizar la salud de sus trabajadores y de las personas a las que prestan apoyo. Teniendo en cuenta que las organizaciones que participaron en el estudio difieren en cuanto 
a número de personas con DID atendidas, recursos, tamaño de la plantilla, etc., los datos obtenidos tras el análisis del impacto económico producido por la situación de alarma ponen de manifiesto la fuerte inversión realizada por el tercer sector para paliar los efectos de la pandemia y el confinamiento.

Dado que la mayor parte de las organizaciones contestaron a la encuesta durante el periodo comprendido entre el 15 de marzo y el 15 de junio, se tiene en cuenta este periodo para calcular el gasto realizado por cada entidad:

- Las organizaciones, en términos promedio, realizaron un gasto semanal de $482,9 €$ en material de protección y otras inversiones necesarias para dar respuesta a las restricciones sanitarias (p. ej., sectorización de espacios), algo que debería haber hecho la Administración pública, siendo el gasto global 359.261 $€$. Solo un $14,5 \%$ de las organizaciones no invirtieron en este concepto.

- Una de cada cuatro entidades no ha realizado ningún gasto en contratación de personal $(41,0 \%)$. En el resto, el gasto en refuerzo de la plantilla asciende, en conjunto, a $958.591 €$.

- El 83,6 \% de las organizaciones han visto mermados sus ingresos anuales, siendo el promedio de ingresos no percibidos de 76.202,6€ por organización y las pérdidas globales de 3.124.306 €.

\subsubsection{Organizaciones y prestación de apoyos}

Según los gerentes y/o responsables, todas las organizaciones que han participado en este estudio, salvo una, han tenido que realizar cambios en sus centros y servicios con el fin de asegurar su buen funcionamiento durante la emergencia sanitaria: cambios en la prestación de apoyos (94,4 \%); cambios que afectaron a los trabajadores $(87,5 \%)$, introduciendo mayor flexibilidad en sus condiciones laborales; y modificaciones en sus espacios (80,6 \%). Sin embargo, a pesar de este esfuerzo, el $60 \%$ de las organizaciones participantes han experimentado dificultades en la prestación de servicios, siendo las más frecuentes: dar los mismos apoyos a las personas con DID (45,2 \%) y sus familias (30,0 \%); ofrecer la adecuada asistencia sanitaria a las personas $(24,7 \%)$; y apoyar a los profesionales que siguieron en activo $(20,5 \%)$. Un 19,2\% de las organizaciones, además, tuvieron que solicitar un ERTE.

A pesar de estas dificultades, casi el $70 \%$ de las organizaciones $(69,3 \%)$ consideran que las personas con DID han recibido la atención necesaria durante la emergencia COVID-19. En el resto, el principal obstáculo en la prestación de apoyos ha sido el cierre de los centros y su escasa flexibilidad para poder seguir ofreciendo los apoyos en otros contextos, seguido de la falta de recursos materiales y humanos y cierta sensación de olvido por parte de la Administración.

Los gerentes y responsables de servicios encuestados consideran que la mayoría de los profesionales se han adaptado bastante bien a la situación, y han valorado positivamente su plena disposición y aceptación de la flexibilización de sus condiciones laborales. Así mismo, el 93,2 \% de los gerentes consideran que la respuesta de su organización ha sido buena o muy buena debido, principalmente, a la rapidez y coordinación 
eficaz para implementar los cambios necesarios, a su capacidad para dar apoyo a las personas con DID y a la plena disposición de los profesionales.

No ocurre lo mismo al valorar la respuesta del Gobierno y las comunidades autónomas, ya que una amplia mayoría de los responsables de los servicios, al igual que hicieron las familias y los profesionales de atención directa, consideran que las medidas tomadas desde la Administración han sido insuficientes $(64,0 \%)$ o inexistentes $(6,7 \%)$. Esta valoración negativa se debe, sobre todo, a la carencia de recursos económicos y material de protección y la ausencia de protocolos y políticas enfocadas al sector de la discapacidad, así como a la falta de una estrategia preventiva y al cierre de servicios esenciales para las personas con DID que ha llevado a la sobrecarga de las familias. Sin embargo, el trabajo con otras entidades locales ha sido valorado positivamente por el $60,0 \%$ de las entidades encuestadas destacando especialmente la colaboración con las fuerzas y cuerpos de seguridad, profesionales sanitarios y bomberos.

\subsubsection{El futuro de las organizaciones del tercer sector}

El 84,0 \% de los gerentes y responsables de servicios que han participado en el estudio consideran que la situación generada por la COVID-19 ha puesto de manifiesto la necesidad de realizar cambios en las organizaciones de las que son responsables, siendo especialmente urgentes los relacionados con la necesidad de innovar en la prestación de apoyos $(90,5 \%)$ para avanzar hacia modelos de trabajo en la comunidad, continuar con la formación y mejora del rendimiento de los equipos profesionales, así como implementar mejores sistemas de evaluación y seguimiento de la prestación de apoyos.

A pesar de percibir que es necesario introducir modificaciones en las organizaciones del tercer sector, el $74,0 \%$ de los responsables participantes consideran posible la continuidad de su organización tal y como ha estado funcionando hasta el momento. Quienes cuestionan esto (26,0 \%) son, en mayor medida, gerentes de organizaciones que, además, llevan muchos años de experiencia en el sector, y lo hacen por tres motivos, principalmente: la necesidad de avanzar hacia procesos de prestación de apoyos en el contexto natural de la persona; la necesaria trasformación tecnológica de las organizaciones y la formación de personas con DID y sus familias en TIC; y los recursos económicos disponibles.

\section{Conclusiones}

La situación de alerta sanitaria provocada por la COVID-19 ha visibilizado e intensificado situaciones de discriminación y exclusión previamente experimentadas por el colectivo de personas con discapacidades intelectuales y del desarrollo (Inclusion Europe, 2020), así como la precariedad del tercer sector, al haber sido necesario un esfuerzo importante para prestar los apoyos necesarios, dados los escasos recursos con que contaba. De manera especial, se ha acentuado la exclusión que los alumnos con DID ya experimentaban en el contexto educativo ordinario (Echeita, 2010), que 
han visto sus apoyos significativamente reducidos. Además, la pandemia ha puesto de manifiesto que una parte importante de la población de personas con DID lleva mucho tiempo confinada, sin poder acceder a apoyos naturales, y con pocas oportunidades para ejercer pleno control sobre su vida (Verdugo y Navas, 2017), lo que lleva a la necesidad de desarrollar y mantener redes naturales de apoyo (Verdugo, 2018).

Antes de presentar las conclusiones, detallamos algunas consideraciones metodológicas que deben tenerse en cuenta a la hora de interpretar (y, sobre todo, generalizar) los datos que se ofrecen en este estudio.

El primer aspecto que debe considerarse es el error muestral. En el caso de la encuesta a personas con DID, teniendo en cuenta los últimos datos recogidos en la Base Estatal de Personas con Discapacidad, la población con discapacidades intelectuales y del desarrollo en nuestro país ascendería a las 274.883 personas. Esto arroja, teniendo en cuenta que 582 personas con DID han participado en el estudio, un $\pm 4 \%$ de error muestral, con un nivel de confianza del $95 \%$. La encuesta a familias presenta un error muestral superior, pues con 323 cuestionarios completados el margen de error se sitúa en torno al $5 \%$. En los cuestionarios dirigidos a organizaciones y profesionales, teniendo en cuenta que la mayor parte de entidades y organizaciones participantes están vinculadas a Plena inclusión, y que esta representa a más de 900 organizaciones y cuenta con 40.000 profesionales, el error muestral ascendería al $\pm 10,7 \%$ para las organizaciones y al $\pm 4 \%$ para los profesionales. Por tanto, los datos que se presentan deben interpretarse como tendencias (no realidades absolutas) de aquello que se pretende estudiar.

La situación de confinamiento nos obligó a realizar una encuesta digital, por lo que aquellas personas con dificultades de acceso a la tecnología o con mayores necesidades de apoyo pueden no estar correctamente representadas, al menos en lo que se refiere a la encuesta a personas con DID. Lo mismo ocurre en aquellas familias, personas, profesionales o gerentes que atravesaban situaciones especialmente delicadas que impedían dedicar tiempo a contestar a un cuestionario. En cualquier caso, no es muy amplia la muestra de hogares que manifiestan tener una mala situación económica, en los cuales las dificultades pueden haberse agravado. Por último, y en relación con la muestra de organizaciones, las entidades participantes presentan una gran variabilidad, tanto en recursos como en tamaño y plantilla, no siendo posible, dado el pequeño tamaño de la muestra, ofrecer una imagen que refleje la situación de cada organización durante la emergencia sanitaria en función de diferencias en las variables comentadas. No obstante, el estudio recoge una gran variedad de situaciones que permite ofrecer una imagen bastante completa del impacto de la COVID-19 y el confinamiento en la vida de las personas con DID, sus familias y las organizaciones y profesionales que les prestaban apoyo.

Por otra parte, conviene matizar que se recomienda cautela en la interpretación de los datos aportados por las organizaciones, pues la muestra de este estudio se reduce a 75 organizaciones y el movimiento asociativo en nuestro país está integrado por más de 1.000 entidades que trabajan con personas con distintas discapacidades intelectuales y del desarrollo. Además, encontramos una importante variabilidad con respecto al tamaño de las organizaciones a las que representan los gerentes y responsables encuestados lo que, sin duda, condicionará algunos de los análisis realizados. 
A continuación, se presentan algunas de las principales conclusiones de este estudio, así como algunas recomendaciones, con el fin de no retroceder en la lucha por la conquista de derechos individuales y para evitar el regreso a un modelo de prestación de servicios exclusivamente "rehabilitador", que considere que para proteger la salud de las personas con DID es necesario prestar apoyos específicos en residencias medicalizadas, alejadas de la comunidad y en las que se sufren confinamientos indiscriminados, con apoyos que dependen del sobreesfuerzo de las organizaciones y sus profesionales. La medicalización de los centros residenciales puede poner en peligro el avance hacia modelos de vida inclusivos. Hay que buscar soluciones por otras vías debiendo ser prioritarias la desinstitucionalización y la transformación de las residencias (Plena inclusión, 2020c).

Es necesario que los servicios de apoyo a personas con DID sean considerados esenciales, pues de ellos depende no solo la calidad de vida de las personas con DID (González, 2019), sino la de sus familias, que se han visto sobrecargadas. Como ya se ha señalado, la no recepción de los apoyos necesarios ha generado consecuencias emocionales importantes en ambos colectivos y también en los profesionales, especialmente en los que trabajaron de manera presencial, lo que lleva a subrayar la necesidad de contar con medidas que, durante el periodo de recuperación de esta crisis sanitaria, no solo refuercen el tercer sector, sino que también lo protejan, garantizando a familias, profesionales y, sobre todo, a personas con DID un adecuado apoyo psicológico.

A pesar de esta situación de desgaste, los datos de este estudio ponen de manifiesto que los profesionales y las organizaciones han intentado no dejar a nadie atrás durante la pandemia, esfuerzo que contrasta con el realizado desde las Administraciones públicas, quienes, según un $60 \%$ de familiares, profesionales y gerentes de organización o responsables de servicios, no han tomado medidas suficientes para proteger a las personas con DID durante la emergencia sanitaria. De ahí la relevancia que tiene generar apoyos mucho más intensos a las familias, las cuales han sido el apoyo fundamental para sus familiares con discapacidad y no tienen un sistema de protección adecuado.

A pesar del esfuerzo realizado por el tercer sector, la crisis sanitaria ha dejado al descubierto las carencias de nuestro sistema de apoyos: un sistema infradotado, que acentúa las situaciones de soledad y exclusión social. El contexto de residencia ha condicionado, además, el tipo de apoyo que las personas con DID han tenido durante el confinamiento, ya que los residentes en contextos específicos parecen haber contado con menos apoyos naturales, y quienes convivieron con su familia han dependido en gran medida de los apoyos proporcionados por esta, siendo anecdótico el apoyo de los centros educativos, ordinarios y especiales. Esta situación lleva a subrayar la necesidad de 'repensar' tres aspectos fundamentales: el grado en que se trabaja en el desarrollo y mantenimiento de redes naturales de apoyo cuando la persona vive en un entorno específico; la medida en que los apoyos ofrecidos desde el tercer sector llegan a otros contextos como el familiar cuando la persona no acude a un servicio concreto; y el papel de nuestro sistema educativo a la hora de garantizar el derecho a la educación cuando existen especiales necesidades de apoyo.

La situación actual debe llevarnos a reclamar el cambio del modelo de apoyos hacia un modelo comunitario inclusivo, que contribuya a mejorar la calidad de vida 
de todas las personas. No se debe correr el riesgo de que el modelo residencial que impera en nuestro país se medicalice aún más. El derecho a la salud no es incompatible con el derecho a vivir una vida significativa, tener un proyecto vital deseado, en el que primen tus seres queridos y apoyos naturales en lugar de fármacos, personal sanitario y grandes instituciones (Navas, 2020). Debemos aprovechar la visibilización que ha provocado la COVID-19 de las carencias de nuestro sistema de atención, muchas veces segregador y con escasos apoyos naturales, para construir hogares en los que todos quisiéramos vivir.

Este estudio presenta algunas limitaciones que debieran tenerse en cuenta. En primer lugar, la situación de confinamiento obligó a realizar una encuesta en línea, por lo que aquellas personas con dificultades de acceso a la tecnología o con mayores necesidades de apoyo pueden no estar correctamente representadas. Tampoco aquellas familias, personas, profesionales o gerentes que atravesaban situaciones especialmente delicadas que impedían dedicar tiempo a contestar a un cuestionario. Una encuesta online tampoco permite 'entrar' en los hogares y centros y detectar situaciones de abuso que hayan podido producirse durante el confinamiento.

Tampoco hemos podido realizar un seguimiento de las consecuencias de la pandemia en población con discapacidades intelectuales y del desarrollo, y consideramos necesario que este seguimiento se realice desde las instituciones públicas por diversos motivos: las personas con discapacidad pueden ser más vulnerables a perder su empleo o su estatus económico en situaciones de recesión, presentan mayores condiciones de salud que pueden no haber sido correctamente atendidas o vigiladas, y han dejado de recibir apoyos que pueden traducirse en una regresión en habilidades previamente adquiridas (Plena inclusión, 2020b). Recordamos, en relación con este último aspecto, que un 78,6 \% de las familias encuestadas muestran preocupación por cómo se adaptará la persona al contexto de 'nueva normalidad' y posible convivencia con el virus; por la continuidad de los apoyos y servicios tal y como han sido conocidos hasta el momento, y por los efectos que la situación de confinamiento y la COVID-19 han tenido o tendrán en la salud de la persona con DID.

Finalmente, los datos reflejan solamente una parte importante de la realidad, descrita a partir del testimonio voluntario de los participantes, y convendría extenderlo a otros centros y organizaciones, como los centros públicos de atención a la dependencia.

La pandemia ha creado una situación de incertidumbre sobre el futuro, ansiedad y estrés para los profesionales, las familias y las personas con DID, que han visto sus vidas alteradas al tener que cambiar sus rutinas diarias y se enfrentan a las consecuencias de un sistema que ha demostrado tener carencias en la prestación de apoyo a las personas con DID en todos los niveles: educación, empleo, salud y servicios sociales. Estas dificultades han dado lugar a la violación de muchos de los derechos de las personas con discapacidad recogidos en la Convención de las Naciones Unidas, como el derecho a la salud, la educación, la accesibilidad o la habilitación y rehabilitación, entre otros. Es, por tanto, urgente adoptar medidas que, en situaciones similares, garanticen que un grupo particularmente vulnerable no sufra una mayor exclusión, y que recojan la realidad específica por la que han atravesado las personas 
IMPACTO DE LA COVID-19 EN LAS PERSONAS CON DISCAPACIDADES INTELECTUALES

Y DEL DESARROLLO, SUS FAMILIARES Y LOS PROFESIONALES Y ORGANIZACIONES DE APOYO

M. CRESPO, M. Á. VERDUGO, P. NAVAS, S. MARTÍNEZ, A. M. AMOR

con DID, sus familias y los profesionales y organizaciones que han prestado apoyo. En definitiva, es el momento de replantearse los servicios y de la transformación organizacional.

\section{Agradecimientos}

El equipo investigador quiere dar las gracias por su colaboración a las casi 1.500 personas que han participado en el estudio en calidad de personas con DID $(\mathrm{N}=582)$, familiares $(\mathrm{N}=323)$, profesionales $(\mathrm{N}=495)$ o responsables de servicios $(\mathrm{N}=75)$. Así mismo, quiere agradecer su implicación en la elaboración de los cuestionarios a Enrique Galván, Javier Tamarit, Berta González, Laura Espejo, Inés de Araoz, Beatriz Vega, Olga Berrios, Antonio Hinojosa, Manuel Posada y al equipo de familias de Plena inclusión España.

\section{Referencias bibliográficas}

Akoglu, H. (2018). User's guide to correlation coefficients. Turkish Journal of Emergency Medicine, 18(3), 91-93. https://doi.org/10.1016/j.tjem.2018.08.001

Amor, A. M., Navas, P., Verdugo, M. Á. y Crespo, M. (2021). Perceptions of people with intellectual and developmental disabilities about COVID-19 in Spain: a cross-sectional study. Journal of Intellectual Disability Research. https://doi.org/10.1111/jir.12821

Baralt, M. (2011). Coding Qualitative Data. En A. Mackey y S. M. Gass (Eds.), Research methods in second language acquisition: a practical guide (pp. 222-244). John Wiley \& Sons.

Beigel, J. H., Tomashek, K. M., Dodd, L. E., Mehta, A. K., Zingman, B. S., Kalil, A. C., ... y Lane, H. C. (2020). Remdesivir for the treatment of Covid-19 - Preliminary report. New England Journal of Medicine, 383(19), 1813-1826. https://doi.org/10.1056/NEJMoa2007764

Courtenay, K. (2020). COVID-19: challenges for people with intellectual disability. The BMJ, 369, m1609. https://doi.org/10.1136/bmj.m1609

Courtenay, K. y Perera, B. (2020). COVID-19 and people with intellectual disability: impacts of a pandemic. Irish Journal of Psychological Medicine, 37(3), 231-236. https://doi. org/10.1017/ipm.2020.45

Echeita, G. (2010). Alejandra L. S. o el dilema de la inclusión educativa en España. En P. Arnaiz, M. ${ }^{a}$ Hurtado y F. J. Soto (Coords.), 25 años de integración escolar en España. Tecnologías e inclusión en el ámbito educativo, laboral y comunitario (pp. 1-13). Consejería de Educación, Formación y Empleo.

Esentürk, O. K. (2020). Parents' perceptions on physical activity for their children with autism spectrum disorders during the novel coronavirus outbreak. International Journal of Developmental Disabilities. Advance online publication. https://doi.org/10.1080/2047386 9.2020 .1769333

European Association of Service Providers for Persons with Disabilities. (2020). The impact of COVID-19 on disability services in Europe. Recuperado de https://www.easpd. eu/sites/default/files/sites/default/files/Publications2020/impact_of_covid-19_on_disability_services_in_europe_a_first_snapshot.pdf

Ediciones Universidad de Salamanca / CC BY-NC-ND

Siglo Cero, vol. 52, número extraordinario, 2021, pp. 11-36 
IMPACTO DE LA COVID-19 EN LAS PERSONAS CON DISCAPACIDADES INTELECTUALES Y DEL DESARROLLO, SUS FAMILIARES Y LOS PROFESIONALES Y ORGANIZACIONES DE APOYO M. CRESPO, M. Á. VERDUGO, P. NAVAS, S. MARTÍNEZ, A. M. AMOR

Folegatti, P. M., Ewer, K. J., Aley, K. P., Angus, B., Becker, S., Belij-Rammerstorfer, S. B., ... y Pollard, A. J. (2020). Safety and immunogenicity of the ChAdOx1 nCoV-19 vaccine against SARS-CoV-2: a preliminary report of a phase 1/2, single-blind, randomized controlled trial. Lancet, 396, 467-478. https://doi.org/10.1016/S0140-6736(20)31604-4

González, J. A. (2019). Intensidad de apoyos, salud mental, empleo y su relación con resultados de calidad de vida. Siglo Cero, 50(2), 73-88. http://dx.doi.org/10.14201/scero20195027388

Hughes, N. y Anderson, G. (2020). The experience of the COVID-19 pandemic in a UK learning disability service: lost in a sea of ever changing variables - a perspective. International Journal of Developmental Disabilities. Advance online publication. https://doi.org/ 10.1080/20473869.2020.1773711

InClusion Europe. (2020). Neglect and discrimination. Multiplied How Covid-19 affected the rights of people with intellectual disabilities and their families. Recuperado de http://www. inclusion-europe.eu/covid-report-2020/

Jeste, S., Hyde, C., Distefano, C., Halladay, A., Ray, S., Porath, M., Wilson, B. y Thurm, A. (2020). Changes in access to educational and healthcare services for individuals with intellectual and developmental disabilities during COVID-19 restrictions. Journal of Intellectual Disability Research, 64(11), 825-833. https://doi.org/10.1111/jir.12776

Naciones Unidas. (2020). COVID-19 and the rights of persons with disabilities: Guidance. Recuperado de https://www.ohchr.org/Documents/Issues/Disability/COVID-19_and_ The_Rights_of_Persons_with_Disabilities.pdf

Navas, P. (2020). Envejecimiento y discapacidad intelectual en tiempos de pandemia: la necesaria revisión de un modelo de servicios para avanzar hacia un modelo de apoyos. https:// futurosingularcordoba.org/2020/06/02/envejecimiento-y-discapacidad-intelectual-entiempos-de-pandemia-la-necesaria-revision-de-un-modelo-de-servicios-para-avanzar-hacia-un-modelo-de-apoyos/

Navas, P., Amor, A. M., Crespo, M., Wolowiec, Z. y Verdugo, M. Á. (2021). Supports for people with intellectual and developmental disabilities during the COVID-19 pandemic from their own perspective. Research in Developmental Disabilities, 108, https://doi. org/10.1016/j.ridd.2020.103813

Navas P., Verdugo, M. Á., Amor, A. M., Crespo, M. y Martínez, S. (2020). COVID-19 y discapacidades intelectuales y del desarrollo: impacto del confinamiento desde la perspectiva de las personas, sus familiares y los profesionales y organizaciones que prestan apoyo. Disponible en https://plenainclusion.org/sites/default/files/00._informe_covid-19_y_discapacidades_intelectuales_y_del_desarrollo.pdf

Navas, P., Verdugo, M. Á., Martínez, S., Sainz, F. y Aza, A. (2017). Derechos y calidad de vida en personas con discapacidad intelectual y mayores necesidades de apoyo [Rights and quality of life of individuals with intellectual disability and extensive support needs]. Siglo Cero, 48(4), 7-66. http://dx.doi.org/10.14201/scero2017484766

O’Cathain, A. y Thomas, K. J. (2004). “Any other comments?” Open questions on questionnaires-a bane or a bonus to research? BMC Medical Research Methodology, 4(1), 25. https://doi.org/10.1186/1471-2288-4-25

Organización Mundial de la Salud. (2020). WHO Director-General's opening remarks at the media briefing on COVID-19. Recuperado de https://www.who.int/dg/speeches/detail/who-director-general-s-opening-remarks-at-the-media-briefing-on-covid19---11-march-2020.

Ediciones Universidad de Salamanca / CC BY-NC-ND

Siglo Cero, vol. 52, número extraordinario, 2021, pp. 11-36 
Plena inclusión. (2020a). Guía de apoyos en casa durante el coronavirus. Recuperado de https://www.plenainclusion.org/sites/default/files/guia_apoyos_en_casa_durante_el_coronavirus.pdf

Plena inclusión. (2020b). El derecho a la educación durante el COVID-19. Análisis, propuestas y retos para la educación del alumnado con discapacidad intelectual o del desarrollo durante el confinamiento. Recuperado de https://www.plenainclusion.org/sites/default/files/ el_derecho_a_la_educacion_durante_el_covid19.pdf

Plena inclusión. (2020c). Perspectiva ética de la desescalada en residencias y viviendas de personas con discapacidad intelectual o del desarrollo. Recuperado de https://www.plenainclusion.org/informate/publicaciones/perspectiva-etica-de-la-desescalada-en-residenciasy-viviendas-de-personas

Pope, C., Ziebland, S. y Mays, N. (2000). Analysing qualitative data. The British Medical Journal, 320(7227), 114-116.

Rose, J., Willner, P., Vooper, V., Langdon, P. E., Murphy, G. H. y Stenfert, B. (2020). The effect on and experience of families with a member who has intellectual and developmental disabilities of the COVID-19 pandemic in the UK: developing an investigation. International Journal of Developmental Disabilities. Advance online publication. https:// doi.org/10.1080/20473869.2020.1764257

SAfta-Zecheria, L. (2020). Challenges posed by COVID-19 to the health of people with disabilities living in residential care facilities in Romania. Disability and Society, 35(5), 837-843. https://doi.org/10.1080/09687599.2020.1754766

Sandelowski, M., Voils, C. I. y Knafl, G. (2009). On quantitizing. Journal of Mixed Methods Research, 3(3), 208-222. https://doi:10.1177/1558689809334210

Schalock, R. L. y Verdugo, M. Á. (2002). Handbook on quality of life for human service practitioners. American Association on Mental Retardation.

Tummers, J., Catal, C., Tobi, H., Tekinerdogan, B. y Leusink, G. (2020). Coronaviruses and people with intellectual disability: an exploratory data analysis. Journal of Intellectual Disability Research, 64(7), 475-481. https://doi.org/10.1111/jir.12730

Verdugo, M. Á. (2018). Conceptos clave que explican los cambios en la provisión de apoyos a las discapacidades intelectuales y del desarrollo en España. Siglo Cero, 49(1), 35-52. https:// dx.doi.org/10.14201/scero20184913552

Verdugo, M. Á. y Navas, P. (2017). Todos somos todos: derechos y calidad de vida de las personas con discapacidad intelectual y mayores necesidades de apoyo. Real Patronato sobre Discapacidad. 